\title{
Evaluación Psicoeducativa y Mediación Pedagógica: Experiencias de integración en Puerto Rico
}

Psychoeducational assessment and pedagogical mediation: Experiences from Puerto Rico

\section{Volumen 20, Número 3}

Setiembre - Diciembre

pp. 1-28

\section{Wanda C. Rodríguez Arocho}

\section{Citar este documento según modelo APA}

Rodríguez Arocho, Wanda C. (2020). Evaluación psicoeducativa y mediación pedagógica: Experiencias de integración en Puerto Rico. Revista Actualidades Investigativas en Educación, 20(3), 1-28. Doi. 10.15517/aie.v20i3.43620 


\title{
Evaluación Psicoeducativa y Mediación Pedagógica: Experiencias de integración en Puerto Rico
}

Psychoeducational assessment and pedagogical mediation: Experiences from Puerto Rico

\author{
Wanda C. Rodríguez Arocho ${ }^{1}$
}

Resumen: Este ensayo se enfoca en la integración de la evaluación psicoeducativa y la mediación pedagógica en el diagnóstico y la intervención de dificultades cognitivas que impactan procesos de aprendizaje. Su objetivo es presentar y examinar ejemplos de dicha integración en estudios realizados en un programa de investigación desarrollado en Puerto Rico a partir del Estudio de Funciones Ejecutivas y Lenguaje (Proyecto EFEL). Tanto dichos estudios como el presente examen adoptan el enfoque histórico-cultural como marco conceptual, en el que los conceptos de mediación instrumental y semiótica, interacción social y comunicación son centrales. Se propone que la mediación pedagógica es una estrategia efectiva para propiciar modificabilidad cognitiva. Para sostener esta proposición se presentan argumentos teóricos vinculados al enfoque histórico-cultural, como los ofrecidos en la Teoría de Inteligencia PASS. También, se examinan herramientas metodológicas como el Cognitive Asssement System y PASS Reading Enhancement Program. Se plantea que se trata de herramientas culturales que permiten enfrentar la visión tradicional de la inteligencia como una propiedad inmutable del sujeto. A partir del examen de resultados de investigaciones realizadas con diversas poblaciones y grupos de edad, se sostiene que el uso de estas herramientas, en la práctica de la mediación pedagógica, la valida como estrategia de educación cognitiva. Se concluye que la mediación pedagógica propicia modificaciones cognitivas que pueden impactar positivamente la ejecución académica y promover mayor equidad y justicia en la valoración del funcionamiento intelectual.

Palabras clave: evaluación psicoeducativa, mediación pedagógica, modificación cognitiva

\begin{abstract}
This essay focuses on the integration of psychoeducational evaluation and pedagogical mediation in the diagnosis and intervention of cognitive difficulties that impact learning processes. Its objective is to present and examine examples of such integration in studies carried out in a research program developed in Puerto Rico originated in the Study of Executive Functions and Language. Both these studies and the present examination adopt the historical-cultural approach as a conceptual framework, in which the concepts of instrumental and semiotic mediation, social interaction and communication are central. It is proposed that pedagogical mediation is an effective strategy to promote cognitive modifiability. To support this proposition, theoretical arguments linked to the historical-cultural approach are presented, such as those offered in the PASS Intelligence Theory. Methodological tools such as the Cognitive Assessment System and PASS Reading Enhancement Program are also examined. It is proposed that these are cultural tools that allow us to confront the traditional vision of intelligence as an immutable property of the subject. From the examination of the results of investigations carried out with different populations and age groups, it is argued that the use of these tools in the practice of pedagogical mediation validates it as a strategy for cognitive education. It is concluded that pedagogical mediation fosters cognitive modifications that can positively impact academic performance and promote greater equity and fairness in the evaluation of intellectual functioning.
\end{abstract}

Key words: psychoeducational evaluation, pedagogic mediation, cognitive modification

\begin{tabular}{l}
\hline 'Catedrática jubilada, Universidad de Puerto Rico, Recinto de Río Piedras, \\
Departamento de Psicología; San Juan, Puerto Rico. Orcid: \\
https://orcid.org/0000-0002-4460-926X
\end{tabular}

Dirección electrónica.wandacr@gmail.com

Ensayo recibido: 12 de marzo, 2020

Enviado a corrección: 11 de junio, 2020

Aprobado: 13 de agosto, 2020 


\section{Introducción}

Es pertinente comenzar por precisar las definiciones de las que parto para facilitar la comprensión de la integración de los temas centrales de este ensayo. El término evaluación psicoeducativa se refiere a "un tipo de evaluación que se utiliza para comprender el funcionamiento cognitivo, académico, social, emocional, conductual, comunicativo y adaptativo de un individuo dentro de un entorno educativo" (Dombrowski, 2015, p. 3). La educación cognitiva es una perspectiva conceptual que plantea que los procesos de enseñanza y aprendizaje pueden orientarse "al desarrollo y el fomento de procesos sistemáticos de percepción, pensamiento, aprendizaje y solución de problemas (Haywood, 2013, p.3.). Es decir, la educación cognitiva busca la modificación de procesos cognitivos.

La mediación pedagógica es una estrategia para la educación cognitiva cuya práctica se origina y desarrolla en enfoques que resaltan la actividad del sujeto en su propio aprendizaje y los procesos caracterizados por la atribución de significado y sentido a la experiencia vivida, la interacción del sujeto con otros actores sociales en el proceso educativo, el diálogo, la comunicación y la colaboración. León León (2014) destaca, entre estos enfoques, las propuestas de Ausubel, Piaget, Vygotski, Bruner y Feuerstein. A partir de estas definiciones se considera la evaluación psicoeducativa como una herramienta diagnóstica cuyos resultados de base a la educación cognitiva mediante estrategias de mediación pedagógica.

La evaluación psicoeducativa y la mediación pedagógica son actividades comunes en los escenarios educativos. Hay diferentes formas de abordarlas teórica y metodológicamente. En este ensayo me aproximo a ellas desde la perspectiva históricocultural, asociada con el trabajo seminal de L.S. Vygotski y las elaboraciones subsiguientes de A. R. Luria. Desde esta perspectiva, ambas son consideradas prácticas sociales institucionalizadas que se realizan con herramientas culturales, se inscriben en premisas filosóficas y construcciones teóricas. El propósito del ensayo es compartir información y reflexiones sobre un programa de investigación desarrollado en Puerto Rico, orientado a propiciar cambios en funcionamiento cognitivo a partir de mediaciones pedagógicas. Para lograr ese propósito, primero discutiré brevemente la justificación científico-técnica de ambas prácticas en tanto generadoras de información, y luego, lo que implica significarlas desde la perspectiva histórico-cultural.

La evaluación del funcionamiento intelectual y del aprovechamiento académico, y la mediación pedagógica son prácticas sociales que se justifican ante la opinión pública como 
productos de investigación científica, lo que generalmente se ha interpretado como conocimiento objetivo, válido, confiable y generalizable. Esta interpretación fue cuestionada desde muy temprano en el desarrollo del enfoque histórico-cultural. Este enfoque postula que la producción y el uso de conocimiento científico son actividades humanas históricamente situadas, culturalmente mediadas y socialmente realizadas (Vygotski, 1991, 1994). Por lo tanto, aproximarse a cualquier tema desde ese enfoque implica analizar los discursos que se producen desde esas coordenadas (Daferemos, 2014). Cada una de ellas aporta algo importante a la comprensión del asunto, en este caso, el tema de la evaluación psicológica en general y de la evaluación psicoeducativa en particular. Aproximarse a la evaluación en clave histórica es útil para apreciar las transformaciones de esos conceptos y esas prácticas, y relacionarlas a cambios en la filosofía y en la sociología de la ciencia y en sus áreas de especialización (Valsiner, 2013).

En el tema que nos ocupa se intersecan dos áreas: psicología y educación. Entender ambos campos de actividad como mediados por las herramientas culturales impone la necesidad de conocer esos artefactos, su origen, su desarrollo, sus aplicaciones y sus implicaciones. Entenderlos como prácticas sociales, por otro lado, obliga a mirar el entramado social e institucional en el que se construye, distribuye y utiliza el conocimiento. Ese entramado implica relaciones dialécticas, intertextuales, intersubjetivas y de poder, aunque este sea un asunto poco discutido explícitamente en la presentación de teorías e investigaciones (Cole, Levitin y Luria, 2006; Dafermos, 2018). En ese entramado los conceptos científicos adquieren valor de herramientas culturales que median pensamientos y acciones. Uno de esos conceptos es el concepto de inteligencia.

Una de las primeras áreas de teorización, investigación y aplicación en la psicología fue la evaluación del funcionamiento cognitivo asociado a la inteligencia. Dazinger (1997) examina el lugar que ese concepto ha ocupado en la historia de la psicología y explica las condiciones históricas, sociales y culturales que le han hecho parte importante del lenguaje de la disciplina. Este autor documenta que, desde el comienzo, la educación es uno de los campos de mayor aplicación. Esto abarca desde el empleo de poblaciones escolares para el desarrollo de pruebas hasta el uso de estas para clasificar y ubicar estudiantes en los espacios escolares. Dazinger (1997) también documenta que la psicometría y la tecnología de pruebas de inteligencia que la acompañó fueron determinantes en mostrar un producto de la psicología que avanzó su reconocimiento y estatus en la comunidad científica y en la sociedad en general. Sin embargo, la historia de la psicología no es lineal y la aproximación 
al concepto de inteligencia y el abordaje a su evaluación se han transformado. Como ocurre en otras ciencias, el desarrollo de conceptos y métodos de estudio ha respondido tanto a cambios histórico-culturales como a las necesidades y exigencias de las sociedades en la que realiza la actividad científica.

Desde sus inicios como ciencia, la psicología asumió una ontología objetivista realista con respecto a sus objetos de estudio. Según Schraw (2013), esta posición plantea la existencia de una realidad independiente del sujeto que la observa, la cual, se supone, tiene propiedades universales. Esto presume que todas las personas la experimentamos y conocemos del mismo modo. Desde esta postura, a la inteligencia se le considera una entidad que se manifiesta de forma clara y distinta. Se trata de una cualidad que las personas poseen. Para producir conocimiento de la inteligencia como objeto de estudio, la psicología optó por una epistemología positivista y abrazó el empirismo como método de estudio (Dazinger, 1997). Esta postura, partió de la premisa de la separación entre objeto y sujeto, se apoyó en reduccionismos, cuantificación y búsqueda de relaciones causales lineales (González Rey, 2009; Guadarrama, 2018).

Cada uno de estos elementos es visible en la teorización y evaluación de la inteligencia, y predominó hasta la década de 1960 cuando dramáticas transformaciones históricas y socioculturales impulsaron cuestionamientos desde la filosofía y la sociología de la ciencia, las cuales llevaron a revisiones profundas en la psicología. Previo a ese viraje, el uso de las pruebas psicométricas para la selección de personal en industrias, para admisión y promoción en centros educativos y para el diagnóstico de un amplio espectro de problemas psicológicos, aumentó exponencialmente en la primera mitad del Siglo XX, y se convirtió en una herramienta cultural mediadora en muchas actividades de la vida social (Dazinger, 1997).

Dadas las premisas ontológicas y epistemológicas desde las que se desarrolló la medición psicológica, la mediación pedagógica no tenía lugar en esa práctica. Es así porque la mediación parte de premisas ontológicas y epistemológicas diferentes y, en consecuencia, lleva a otras prácticas de estudio e intervención. Según la clasificación de Schraw (2013), su ontología sería constructivista y se vincularía al contextualismo. De acuerdo con este autor, la ontología constructivista rechaza la idea de la existencia de realidades independientes del sujeto y propone que las personas negociamos y consensuamos enterados de nuestros contextos particulares de actividad. Desde esta perspectiva, la inteligencia no es una propiedad del sujeto, sino una propiedad que emerge de la relación entre el sujeto y la 
situación en que su accionar será juzgado como inteligente. La producción de conocimiento, desde este nuevo ángulo, demanda una epistemología interpretativa asentada en la complejidad (Guadarrama, 2018).

En el desarrollo de la perspectiva histórico-cultural, Vygotski (1991) partió de un análisis profundo de la psicología de su época que le permitió elaborar una perspectiva para entender la forma en que la ciencia y sus aplicaciones eran atravesadas por dinámicas históricas, sociales y culturales. Hizo críticas al positivismo y al empirismo que parecen más de nuestra época que de la suya (Dafermos, 2014). Planteó la importancia de enfocar procesos y movimientos en el desarrollo intelectual más que sus productos acabados, y de considerar que la afectividad, la motivación y la intencionalidad atraviesan los procesos intelectuales. Argumentó que el ambiente no afecta de forma directa y unilateral la acción de un sujeto en la solución de un problema o la realización de una tarea, sino que es su vivencia, su atribución de sentido subjetivo a la situación, la que explica el comportamiento (Rodríguez Arocho, 2011). Por tanto, la situación social del desarrollo y una perspectiva compleja de las zonas de desarrollo próximo en que transcurre la vida son esenciales para una evaluación comprensiva y justa de su funcionamiento (Rodríguez Arocho, 2015).

También elaboró el argumento de que es una falacia creer que los datos están al margen de las teorías. Elaboró el argumento sobre la base de que toda observación se hace desde un lugar que condiciona la mirada del observador e impone límites a lo que se observa y a los métodos de evaluación que considera válidos. Es decir, nuestra mirada se enfoca a encontrar lo que buscamos. Al poner énfasis en la teoría y en los supuestos ontológicos y epistemológicos que las subyacen, Vygotski confrontó el entendido tradicional de ciencia e invitó al cuestionamiento de las premisas de las que partimos al evaluar e investigar, y a tomar conciencia de sus consecuencias.

Las reflexiones de Vygotski con respecto a la forma en la se produce la ciencia y se utiliza destacaron el énfasis desmedido en la dimensión cuantitativa y los resultados en el estudio de los fenómenos, en detrimento de la dimensión cualitativa y los procesos, así como su insistencia en una aproximación sistémica, fueron hechas entre 1924 y 1934. Impresiona notar la forma en que estas críticas se reflejan hoy en el campo de la evaluación psicoeducativa. Una tendencia contemporánea en ese campo apunta hacia un énfasis en la fundamentación teórica de los instrumentos de evaluación, bajo el argumento de que ello facilita su inteligibilidad, así como el análisis, interpretación y comunicación de resultados (Andrews, Saklofske y Janzen, 2001). 
Otra tendencia es el interés por desarrollar instrumentos que permitan una aproximación a procesos cognitivos y neuropsicológicos, lo que implica un viraje con respecto a la visión de la inteligencia como entidad unidimensional, universal, expresada en una habilidad general indiferenciable. Ese viraje ha sido impulsado tanto por investigaciones que subrayan gran variedad de habilidades intelectuales entre sujetos, así como por la relación de esa variabilidad con condiciones socioeconómicas, de raza y género, lo que subraya su enclave sociocultural. También, es tendencia la consolidación de la evaluación dinámica o avalúo, en cuya práctica la evaluación y la intervención se entrelazan. Esta perspectiva, se inscribe en la idea de que el aprendizaje bien orientado puede promover el desarrollo, se enfoca más en el potencial de aprendizaje de los sujetos y en su movilización que en obtener un valor estático de ejecución (Daniels, 2001). Este enfoque, que desde sus inicios se asoció con las ideas de Vygotski sobre la mediación instrumental y simbólica en la actividad humana, la zona de desarrollo próximo y la relación aprendizaje-desarrollo, se ha complejizado con el tiempo (Kozulin, 2014). Varias décadas de práctica e investigación en el campo de la evaluación dinámica y en el acceso a textos inéditos de Vygotski y su discusión en el contexto de lo que se ha denominado una revolución revisionista (Yasnitsky, Van der Veer, Aguilar y García, 2016) han permitido profundizar al respecto.

A partir de las ideas de Vygotski, Luria desarrolló un programa de investigación en neuropsicología que fue fundamental para avanzar las ideas de plasticidad cerebral y modificabilidad cognitiva por vía de la educación (Akutina, 2003). Su trabajo ha sido fundamental en el desarrollo de nuevas miradas a la inteligencia, su evaluación y el desarrollo de mediaciones pedagógicas. En conjunto, elaboraron una mirada a la relación aprendizaje-desarrollo que, en su momento, permitió el diseño de estrategias educativas para aumentar las posibilidades de desarrollo de personas con limitaciones físicas y mentales, y que ha sido fundamento de elaboraciones contemporáneas vanguardistas en la educación especial, así como en la educación regular. La integración del trabajo de Vygotski y de Luria es la base de la mediación pedagógica en clave histórico-cultural.

\section{Desarrollo del tema}

La tesis central del enfoque histórico-cultural es que la conciencia humana se forma y transforma en el transcurso de actividades que ocurren en contextos históricos particulares, son mediadas por las herramientas culturales disponibles y se realizan en prácticas sociales. Al nacer, la cría humana viene a un mundo de objetos materiales, signos y símbolos a los 
que accede, y aprende a utilizar en virtud de su relación con las personas que comparten ese mundo. Por ello, para Vygotski el hecho fundamental de la psicología es la mediación (Moll, 1990). La centralidad de la noción en el enfoque histórico-cultural fue destacada por Werstch (1991) cuando hizo notar que las herramientas e instrumentos mediadores dan forma esencial a la actividad. Es imposible explicar la actividad humana sin la consideración de las herramientas que utiliza para realizarla. Esto hace del tema de la mediación cultural uno central en el enfoque histórico-cultural.

En el desarrollo del concepto de mediación, Vygotski diferenció entre dos tipos de herramientas creadas en el curso del desarrollo cultural de la especie: herramientas materiales para orientar la actividad externa del sujeto en su medio ambiente y herramientas simbólicas para orientar la actividad interna o psíquica (Vygotski, 1931; Vygotski y Luria, 1930). Con respecto al tema que nos ocupa, en la primera categoría pueden ubicarse las pruebas psicológicas y en la segunda, la representación y el manejo mental que hacemos de ellas en virtud de nuestro dominio del lenguaje y de otros sistemas simbólicos en nuestro entorno. Aunque Vygotski visualizó una relación dialéctica entre ellas, no pudo evitar que, con la distinción, se perpetuara la dicotomía entre lo externo y lo interno, que en sus últimos trabajos intentó superar. En el esfuerzo por superar el problema de la dicotomía y complejizar el asunto, Cole (1996) propuso la noción de artefacto como una de mayor utilidad para explicar la mediación cultural en el desarrollo.

En una elaboración de la tesis de mediación cultural, Cole (1996) plantea que el concepto de artefacto tiene mayor alcance que el de herramienta, utilizado por Vygotski y Luria, que era el común en su época. Este autor argumenta que la noción de artefacto tiene un carácter genérico, mientras que la de instrumento o herramienta tiene un carácter más específico. Considera la noción de artefacto como útil para superar la escisión entre lo material y lo ideal, entre lo objetivo y lo subjetivo. Esta escisión es evidente en la propuesta de Vygotski en la que las herramientas materiales orientan la actividad externa y los signos la actividad interna.

Para Cole (1996), el artefacto es la materialización de una idea que se concibe con el objetivo de dar forma a la actividad que se realiza y lograr efectivamente su propósito. Propone una organización jerárquica progresivamente compleja de los artefactos. Los artefactos primarios son los utilizados directamente en una producción y son materia transformada por la acción humana, como los instrumentos de escritura y las tecnologías de comunicación. 
Los artefactos secundarios, por otra parte, son modos de representación de los artefactos primarios y modos de acción en su uso compartido por grupos sociales. Se les considera fundamentales en la transmisión de creencias y modos de actuar tanto a nivel individual como colectivo. Finalmente, los artefactos terciaros consisten en un imaginario que se forja en el proceso de uso y en las dinámicas socioculturales que produce. En este nivel se han producido transformaciones mentales que implican cambios en las relaciones entre sujetos y objetos (interacción humanos-dispositivos) y entre sujetos (intersubjetividad e interacciones sociales). Rodríguez Arocho (2018) ha utilizado las nociones de herramientas y artefactos para analizar esas transformaciones y discutir sus implicaciones para la educación. Para esta autora, el concepto de herramientas o instrumentos utilizado por Vygotski y Luria buscaba explicar la configuración de la mente individual. El concepto de artefacto propuesto por Cole, por otro lado, busca explicar las dinámicas socioculturales que subyacen a esa configuración y resalta el tema de la mediación cultural.

El concepto de mediación cultural es central en la idea de la sociogénesis de la conciencia propuesta por Vygotski. Su formulación más clara se encuentra su ley genética del desarrollo, también conocida como la ley de doble formación. El concepto de mediación pedagógica es un derivado lógico de esta proposición. Kozulin (2003) examina cómo el aprendizaje mediado, que implica mediación pedagógica, deriva de las ideas de Vygotski. Específicamente, del planteamiento de que el desarrollo de funciones mentales superiores, entre las que incluye la atención voluntaria, la memoria lógica, el razonamiento y el ejercicio de la voluntad, se inicia en interacciones de la cría humana con la gente en su mundo. Eventualmente, esas funciones se transforman en intrapsicológicas. Sin embargo, a pesar de las simplificaciones que se han hecho de su trabajo, el desarrollo de las funciones intrapsicológicas no es un proceso automático de copia. En el proceso de apropiación y domino de las funciones se generan transformaciones en ellas que se corresponden con la situación social de desarrollo del sujeto y sus vivencias. Si bien se trata de un proceso que cada sujeto realiza, Vygotski y Luria consideraron que la educación podía influirlo.

Luria fue clave en realizar investigaciones que apoyaron esta propuesta de varias formas. En primer lugar, su investigación gira en torno a cómo la alfabetización media el desarrollo de funciones cognitivas complejas, como la percepción, la atención voluntaria, la memoria lógica y el pensamiento abstracto (Luria, 1976). En segundo lugar, sus investigaciones neuropsicológicas le llevaron a concluir que la mediación pedagógica podía ser una herramienta fundamental para promover rehabilitación y modificación cognitiva en 
personas con lesiones cerebrales de diverso tipo (Luria, 1973). La idea de que la mediación pedagógica podía impulsar reorganización de funciones cerebrales y mejorar la ejecución en pruebas neuropsicológicas es inconcebible desde modelos que entienden el funcionamiento cognitivo complejo como predeterminado, inmutable y universal. La investigación neuropsicológica contemporánea avanza sobre el legado de Luria y la perspectiva históricocultural (Ardila, 2018).

Las ideas y prácticas de la evaluación psicoeducativa y la mediación pedagógica en clave histórico-cultural están implícitas en el concepto de zona de desarrollo próximo. La evaluación tradicional se enfoca en lo que el sujeto es capaz de hacer solo y busca una medida de capacidad actual. A la luz de la mediación, el interés se mueve hacia lo que el sujeto es capaz de hacer con la ayuda de otros en contextos de actividad. No es la capacidad actual lo que se busca estimar, sino el potencial de desarrollo y las formas de estimularlo. La zona de desarrollo próximo no es una propiedad del sujeto, sino un espacio de mediación pedagógica atravesado por la situación social de desarrollo, las vivencias, los artefactos disponibles y las relaciones de colaboración que se establecen (Rodríguez Arocho, 2015).

Los vínculos entre mediación pedagógica, el desarrollo de procesos mentales, particularmente los procesos cognitivos asociados a la conducta inteligente, y las formas de evaluar dichos procesos han generado aplicaciones interesantes. En este caso, la mediación pedagógica se caracteriza porque no separa las acciones didácticas para facilitar el aprendizaje de contenidos, de la forma en cómo esas acciones afectan el desarrollo de funciones mentales específicas como atención voluntaria, memoria lógica, razonamiento y solución de problemas. Se apoya en interacciones interpersonales y comunicación, derivados del trabajo de Luria y Vygotski, sobre el rol del lenguaje en el funcionamiento intelectual (Vygotski, 1934; Luria, 1961,1982). La mediación pedagógica tampoco limita la acción educativa a impactar sobre las evaluaciones en contextos escolares, sino que permite relacionarla con la evaluación y su valor formativo (Rodríguez Arocho, 2011).

\subsection{Proposición}

A partir de lo expuesto, sostengo que, como práctica social, la evaluación psicoeducativa puede optimizar el desarrollo cognitivo y los aprendizajes escolares si utiliza herramientas culturales efectivas como mediadoras del aprendizaje en lugar de usarlas solo como medidas de su resultado. Esta proposición se sostiene en desarrollos teóricos y 
metodológicos en la perspectiva histórico-cultural. Daniels (2001), Kozulin (2000, 2003, 2014) y Moll (2014) son algunos autores que se han ocupado de analizar las contribuciones de Vygotski a la comprensión de la relación entre pedagogía y desarrollo humano. Este último ha examinado el aprendizaje mediado y la evaluación dinámica en detalle. Plantea que la mediación pedagógica debe trascender la enseñanza de contenidos y enfocarse en la enseñanza de instrumentos psicológicos que promuevan funciones cognitivas. Define estos como recursos simbólicos, entre los que menciona signos, símbolos, fórmulas y medios gráficos que ayudan al sujeto a regular procesos cognitivos como los antes mencionados.

Las estrategias para recordar y recuperar información son creadas por la cultura. Ese es el caso de los monumentos, los archivos históricos y la memoria en dispositivos tecnológicos. El uso y dominio de estos instrumentos permite formas más complejas de atender, recordar, planificar y procesar información del medio ambiente. En el proceso de apropiación de esos instrumentos, estos se transforman en herramientas psicológicas que orientan y regulan la actividad del sujeto e interacción con sus motivos, deseos y necesidades. Este planteamiento y la investigación que lo apoya ha dado paso al desarrollo de programas de educación cognitiva y a la construcción de instrumentos de evaluación enfocados en procesos.

Es pertinente hacer notar que, aunque las aplicaciones contemporáneas se orientan principalmente a la educación regular, el interés de Vygotski y de Luria se desarrolló originalmente estudiando el funcionamiento de personas con limitaciones sensoriales (ceguera y sordera) y cognitivas (retraso mental, afasias). Sus investigaciones los llevaron a concluir que las personas con limitaciones como estas podían beneficiarse de mediación pedagógica si en lugar de enfocar la intervención en las deficiencias de los sujetos se enfocaba en sus fortalezas. El desarrollo de estas personas puede verse comprometido cuando se utilizan formas tradicionales de evaluación, que dan una medida fija de cociente intelectual o ejecución en una tarea, y se utiliza ese resultado como criterio principal para el desarrollo de intervenciones limitando el desarrollo de capacidades potenciales (Daniels y Hedegaard, 2011).

Mitjáns Martínez (2009) examina las ideas de Vygotski al respecto, las cuales, posteriormente, Luria integró a sus proyectos de rehabilitación neuropsicológica. Cinco ideas resaltan. Primero, la idea de defecto primario (de naturaleza biológica) y defecto secundario (de naturaleza social). El primario solo se convierte en deficiencia si el contexto cultural y las prácticas sociales no ofrecen los medios para superarlo. Segundo, lo anterior implica que hay 
una construcción social de la deficiencia y que, siendo así, es posible alterarla mediante la toma de conciencia y la acción correspondiente a nivel sociocultural. Tercero, con esa modificación, la educación se enfoca en el sujeto integral y no en un defecto particular. Cuarto, lo anterior presupone que el sujeto que muestra una debilidad en su desarrollo también posee fortalezas que pueden movilizar procesos compensatorios y potenciar transformaciones. Quinto, ese sujeto tiene también un potencial creativo y puede generar sus propios modos de manejo para construir y reconstruir su persona.

En estas cinco ideas se reconoce que el sujeto particular tiene capacidades y potencialidades que pueden ser impulsadas en su medio ambiente social. La educación efectivamente organizada ofrece al sujeto oportunidades para el acceso, apropiación y dominio de formas de autorregulación del propio comportamiento. Dicha organización debe reconocer la dinámica entre lo social y lo individual y entre lo cognitivo y lo afectivo. Lo dicho tiene implicaciones importantes para la evaluación de personas con deficiencias y la mediación pedagógica para promover su desarrollo. Al mismo tiempo, sincroniza muy bien con las nuevas corrientes en el enfoque histórico-cultural que buscan el rescate de conceptos que quedaron al margen las lecturas previas de Vygotski, como el sentido, la vivencia, las emociones y la situación social del desarrollo.

\subsection{Argumentos para la discusión}

La perspectiva elaborada por Luria y Vygotski también ha dado paso a una serie de aplicaciones teóricas y metodológicas que facilitan la argumentación de la proposición expresada. Algunos de los aspectos examinados de esta perspectiva fueron utilizados en la formulación de la teoría de inteligencia PASS (por las siglas en inglés para planificación, atención, procesamiento simultáneo y procesamiento sucesivo de información). Dicha teoría es el resultado de la integración de la propuesta de Luria sobre el funcionamiento cerebral, los desarrollos en las neurociencias contemporáneas y las investigaciones generadas en el campo de procesamiento de información. Su formulación más completa fue publicada por Das, Naglieri y Kirby (1997). Dicha teoría se ha utilizado en dos campos de actividad: la evaluación psicoeducativa y el desarrollo de programas de educación cognitiva o mediación pedagógica. La primera se observa en el Cognitive Assessement System (Naglieri y Das, 1997). La segunda se observa en un programa para el mejoramiento de problemas de lectura llamado PREP (PASS Reading Enhancement Program), el cual fue desarrollado por Das (2000). También, se observa en un manual de intervenciones con estrategias de 
mediación pedagógica orientado a padres, madres y docentes (Naglieri y Pickering, 2010). Describiré, brevemente, estas aplicaciones porque fueron las utilizadas en las investigaciones que discutiré en la próxima sección. Paso a examinar cada una y su uso en investigaciones en Puerto Rico.

La Teoría de Inteligencia PASS. En el contexto de nuevas concepciones de la inteligencia (Bermonti-Pérez, Díaz-Flores, Moreno Torres y Rodríguez Arocho, 2014), se construye la Teoría de Inteligencia PASS (Das, Naglieri y Kirby, 1994). Esta teoría se apoya en la conceptualización de Luria (1973) del cerebro como un sistema funcional. El concepto de función en su modelo es importante porque remite a acciones que realiza el sujeto para recibir y procesar estímulos e información, y orientar su conducta. Luria había trabajado con Vygotski en la investigación del desarrollo de funciones mentales superiores en diversas poblaciones. Tras el fallecimiento de Vygotski, Luria enfocó su trabajo en la neuropsicología desde una perspectiva histórico-cultural. Sus investigaciones en ese campo le llevaron a concluir que el cerebro humano es un sistema compuesto por tres unidades, cada una con funciones específicas, pero relacionadas entre sí. La primera unidad regula la excitación y la atención; la segunda unidad se ocupa de codificar la información mediante procesamiento sucesivo y procesamiento simultáneo; y la tercera unidad se ocupa de la planificación, el automonitoreo y la organización de actividades cognitivas. Para las últimas dos unidades, Luria identificó áreas primarias, secundarias y terciarias en la actividad cerebral, las cuales reflejan niveles de creciente complejidad en el manejo e integración de información. Demostró la capacidad del cerebro de modificar funciones y reorganizarse en función de experiencias de aprendizaje y modificaciones ambientales. La investigación neuropsicológica contemporánea reconoce su deuda con el legado de Luria, al tiempo que elabora sobre el mismo tema (Ardila, 2018).

La teoría del cerebro en acción, nombre por el que se conoce la teoría de Luria (1973), y las investigaciones posteriores en el área de procesamiento de información, son la base de la Teoría de Inteligencia PASS, postulada por Das, Naglieri y Kirby (1994). Esta teoría se contrapone a la noción tradicional de la inteligencia como la expresión de una habilidad o factor general. En ella, la inteligencia se conceptúa como multidimensional. El planteamiento central es que la acción inteligente es el resultado de un conjunto de procesos cognoscitivos diferenciados, pero interdependientes. Propone cuatro procesos básicos en este conjunto. Estos son: planificación, atención, procesamiento sucesivo y procesamiento simultáneo. Son 
estos procesos los que dan nombre a la teoría PASS. Como puede notarse, estos procesos se corresponden con la actividad de las unidades funcionales propuestas por Luria.

Desde su aparición, la Teoría de inteligencia PASS ha generado un cuerpo importante de investigación del que se derivó un instrumento de evaluación cuyo proceso de construcción, validación, usos y estudios de efectividad se documenta en compendios en torno al estado actual de la evaluación intelectual publicados en Estados Unidos de Norteamérica (Naglieri, Das y Goldstein, 2012; Naglieri y Otero, 2011).

Cognitive Assessment System (CAS). Este es un instrumento diseñado por Naglieri y Das (1997) para evaluar el funcionamiento cognoscitivo con base en la teoría PASS. EI CAS es una prueba que tiene cuatro escalas, a su vez divididas en tres subescalas. Las tareas en las subescalas evalúan las actividades que definen cada proceso básico. La planificación es definida como un proceso mental mediante el cual la persona determina, selecciona y utiliza estrategias efectivas para la solución de problemas. Involucra formación de representaciones mentales, solución de problemas, control de impulso, control de procesamiento y recuperación de información.

La atención, por su parte, es definida como un proceso que permite atender selectivamente algunos estímulos al tiempo que se ignora otros. Implica actividad cognitiva enfocada, y es un elemento clave en la resistencia a la distracción, la orientación de la conducta y la vigilancia. El procesamiento simultáneo integra los estímulos en grupos, lo que permite verlos como una totalidad en relación con sus partes. Implica actividad de integración con respecto a estímulos tanto verbales como no verbales. El procesamiento sucesivo, por otro lado, es la actividad que se realiza sobre la información para integrar estímulos verbales y no verbales en un orden serial específico. En su conjunto, la prueba está enfocada a evaluar las estrategias utilizadas para solucionar las tareas cognitivas y para presentar y facilitar la comprensión de la naturaleza de los errores.

A partir de investigaciones sobre el uso del instrumento, este fue revisado y ahora se conoce como CAS-2 (Naglieri, Das y Goldstein, 2012). Esta revisión cuenta con una traducción al español (Naglieri, Moreno y Otero, 2016). Esta traducción le sigue a una que fue realizada mediante autorización de la casa editora, con fines de investigación en el año 2000 (Rodríguez Arocho, 2003). El instrumento ha sido utilizado como sistema de evaluación con poblaciones hispanas en los Estados Unidos de Norteamérica, y se ha documentado su efectividad en contextos escolares (Naglieri y Otero 2018ab). 
Una particularidad de este sistema de evaluación es que fue diseñado para medir funciones cognitivas importantes en la conducta inteligente. El sistema facilita una amplia gama de diagnósticos diferenciales y la planificación educativa en sujetos de 5 a 18 años. La mediación pedagógica y las intervenciones para la modificación cognitiva son centrales en dicha planificación.

La batería principal incluye dos subpruebas de cada una de las cuatro escalas PASS. Cada escala PASS, así como la escala completa CAS2, produce una puntuación estándar con una media de 100 y una desviación estándar de 15. La batería extendida CAS2 tiene tres subpruebas en cada una de las cuatro escalas PASS. Para evaluar planificación se aplican tres subpruebas. Estas son: códigos planificados, conexiones planificadas y coincidencia de números planificados. Estas subpruebas requieren determinar, seleccionar y usar una estrategia para resolver un problema. Para evaluar atención, proceso que requiere mantener el foco en un estímulo particular y evitar la distracción por estímulos competitivos, se utilizan las subpruebas de: atención expresiva, detección de números y atención receptiva. El procesamiento simultáneo de información, que implica la integración de estímulos separados en un solo conjunto, es evaluado con las subpruebas de matrices, relaciones verbales-espaciales y memoria de figuras. El procesamiento sucesivo, que demanda el ordenamiento en serie, es evaluado con las subpruebas: serie de palabras, repetición de oraciones (de 5 a 7 años) o preguntas de oraciones (de 8 a 18 años) y alcance visual de dígitos.

El CAS-2 produce, además, seis puntajes compuestos suplementarios: función ejecutiva sin memoria de trabajo, función ejecutiva con memoria de trabajo, memoria de trabajo, contenido verbal, contenido no verbal y velocidad / fluidez. También se proporciona una comparación visual versus auditiva. Naglieri y Otero (2017) publicaron un libro orientado a explicar el instrumento y su sostén teórico e investigativos, y promover y facilitar su uso. Dicho uso ha producido un cuerpo de investigación con diversas poblaciones, lo cual se ha documentado en la literatura (Naglieri, Das y Goldstein, 2012; Naglieri y Otero, 2108ab).

PASS Reading Enhancement Program (PREP). Este es un programa de intervención remedial para niños y niñas con problemas en la lectura. Como indica su nombre, se fundamenta en la Teoría PASS. Fue desarrollado por J. P. Das y sus colaboradores en la Universidad de Alberta en Canadá (Das, 2000). Este programa es el resultado de la integración de la Teoría PASS y las ideas de Vygotski sobre la zona de desarrollo próximo como un espacio en el que la enseñanza, el aprendizaje y la interacción colaborativa juegan 
un rol central (Das, 2000). El programa hace énfasis en esa interacción y hace de la mediación educativa un proceso de colaboración para el desarrollo de estrategias en la solución de problemas. La mediación se orienta a actividades que faciliten transferir las estrategias aprendidas de una situación a otra para la solución efectiva de problemas diferentes.

El programa consta de diez tareas que varían considerablemente en contenido y en los requisitos que debe realizar el niño o la niña. A pesar de la variedad, el conjunto está orientado a las funciones de procesamiento simultáneo y sucesivo, por ser las más claramente vinculadas a la lectura en la literatura. Debe recordarse, sin embargo, que las funciones se relacionan y son interdependientes. Cada tarea involucra tanto un componente de capacitación global como un componente llamado puente, que se relaciona con el plan de estudios al momento de las tareas. El componente global incluye tareas estructuradas, no lectoras, que requieren la aplicación de estrategias de procesamiento simultáneo y sucesivo. Estas tareas están diseñadas para ofrecer la oportunidad a los niños y a las niñas de internalizar estrategias a su manera, lo que debe facilitar la transferencia. El componente de puente, por otro lado, implica las mismas demandas cognitivas que su componente global combinado y enfatiza tareas de procesamiento simultáneas y sucesivas que se han relacionado en la investigación con el dominio de la lectura y la escritura.

Las tareas globales comienzan con contenido que es familiar y no intimidante, de modo que la adquisición de la estrategia se produzca en pequeñas etapas, casi a manera de juego. La complejidad se introduce paulatina y únicamente después de volver a un contenido que resulta de fácil manejo. La mediación verbal entre el tutor o tutora y el niño y la niña es central. A través de ella, que ocurre mediante discusiones específicas de las estrategias utilizadas, los componentes globales y puente de PREP se utilizan para motivar a la niñez a aplicar sus estrategias a tareas académicas, como la decodificación de palabras. Los componentes global y puente, se dividen en tres niveles de dificultad.

Esta mediación se integra en un sistema de avisos para cada componente global y puente. La serie de avisos crea una red de andamios que sirven de apoyo y orientación para garantizar que las tareas se completen con una cantidad mínima de asistencia y una cantidad máxima de éxito. Un registro de estas indicaciones proporciona un sistema de monitoreo para que el profesorado determine cuándo el material es demasiado difícil para un niño o niña o cuándo un niño o niña puede progresar con éxito a un nivel más difícil. Se requiere un criterio de $80 \%$ de respuestas correctas antes de que un niño o niña pueda pasar 
al siguiente nivel de dificultad. Si no se cumple este criterio se utiliza un conjunto alternativo de tareas, en el mismo nivel de dificultad, para proporcionar la preparación adicional requerida. Las tareas están diseñadas para desarrollar estrategias cognitivas, como ensayo, categorización, monitoreo del desempeño, predicción, revisión de predicción, sondeo y combinación de sonidos. El PREP fue traducido y adaptado al español en España por Garrido Laparte (1999), quien le llamó Programa para la Reeducación del Aprendizaje. Esta versión ha sido utilizada en investigaciones en España y luego adaptada para uso en Puerto Rico (Báez, 2019; Medina, 2018).

Helping children learn: Interventions handhouts for use in school and at hom. Esta es otra herramienta de mediación pedagógica en la forma de un manual, que ofrece 75 intervenciones que permiten la mediación educativa en la escuela y en el hogar (Naglieri y Pickering, 2010). Las intervenciones están orientadas a fortalecer el desarrollo de planificación, atención, procesamiento sucesivo y procesamiento simultáneo siguiendo la Teoría PASS. Se incluye un cuestionario que permite al psicólogo o psicóloga escolar identificar debilidades, fortalezas y necesidades del estudiante para el mejoramiento de su ejecución escolar. El profesorado utiliza las intervenciones que atienden dichas necesidades. Las intervenciones buscan el desarrollo y fortalecimiento de destrezas de atención, memoria, razonamiento y autocontrol, las cuales se consideran necesarias para la realización efectiva de tareas académicas como lectura, escritura y cálculos matemáticos.

La segunda edición ofrece versiones en español para algunas de estas intervenciones, realizadas por Mary A. Moreno Torres y Tulio Otero (Naglieri y Pickering, 2010). Hay dos características de este trabajo que reflejan tendencias sociales en los Estados Unidos de Norteamérica. La primera tiene que ver con el interés de mejorar las oportunidades de aprovechamiento académico en grupos minoritarios, particularmente niños y niñas hispanohablantes. La segunda característica es que el trabajo realizado responde al interés de empoderar docentes, madres y padres mediante la transferencia de conocimiento tradicionalmente limitado a especialistas. Estas dos características se inscriben en un movimiento que busca equidad y justicia en la evaluación psicoeducativa realizada con menores de edad en condiciones de desventaja social (Sepúlveda-Miranda, Otero y Moreno Torres, 2018).

De la teoría y la instrumentación a su aplicación: Producciones investigativas. El trabajo de investigación con la Teoría PASS, el CAS y el PREP tiene una historia en Puerto Rico que cubre 20 años y una prehistoria que abarca seis (Rodríguez Arocho, 2006; 
Rodríguez Arocho y Moreno Torres, 2018). Es una muestra de trabajo colaborativo en comunidades de aprendizaje y de práctica que han sido generadoras de actividad investigativa, docente y profesional comprometida socialmente. El trabajo realizado está representado en una sección especial de la Revista Puertorriqueña de Psicología publicada en dos volúmenes recientes. El volumen 29 (2018) incluye un prefacio por Jack A. Naglieri, una introducción de Rodríguez Arocho y Moreno Torres, y ocho investigaciones en las que se utilizan la Teoría PASS y el CAS. El volumen 30 (2019) incluye una traducción de la introducción al idioma inglés y tres artículos que reportan otras investigaciones. Antes de pasar a examinar con cierto detalle los artículos específicamente relacionados con mediación pedagógica procede una breve nota histórica para poner en perspectiva el trabajo realizado.

En 1994 coincidí con J.P. Das y Jack Naglieri en Moscú en un congreso internacional dedicado al legado de Vygotski y su desarrollo (Rodríguez Arocho y Moreno Torres, 2018). Era un congreso relativamente pequeño que se llevó a cabo en un centro de convenciones en la periferia de la cuidad en donde, además de participar en conferencias y ponencias compartíamos comidas, paseos e interesantes tertulias. El libro sobre la Teoría PASS acababa de salir de imprenta y en la despedida, Naglieri me regaló una copia. Cinco años después concebí una investigación para estudiar la relación entre el desarrollo de lenguaje y funciones ejecutivas en niños y niñas diagnosticados con el Trastorno de Déficit de Atención e Hiperactividad, enmarcada en las contribuciones de Vygotski y Luria, resultaba lógico pensar en modos de evaluar afines al marco conceptual. Retomé el contacto con Naglieri. Desde el primer momento su respaldo fue entusiasta, decidido y permitió vínculos de colaboración perdurables (Rodríguez Arocho y Moreno Torres, 2018).

El Estudio de Funciones Ejecutivas y Lenguaje (Proyecto EFEL) se realizó de 2000 a 2005 en el entonces Centro Universitario de Servicios y Estudios de Psicología de la Universidad de Puerto Rico, hoy Instituto de Psicología (Rodríguez Arocho, 2003, 2004). Este proyecto era uno de cinco dirigidos por miembros de la facultad del Departamento de Psicología que simultáneamente participábamos de un programa de formación en investigación con el apoyo de pares con mayor experiencia en esa actividad. Tanto el programa de adiestramiento como los proyectos individuales recibieron financiamiento del Instituto Nacional de Salud Mental de Estados Unidos de Norteamérica y de la Universidad de Puerto Rico. El financiamiento permitió el reclutamiento de una coordinadora y asistentes de investigación de postgrado a nivel de maestría y doctorado, a los que se sumaban estudiantes de pregrado en prácticas supervisadas y estudiantes de escuela secundaria 
pertenecientes a un programa de formación temprana en investigación (Rodríguez Arocho y Alom Alemán, 2009). Esto creó las condiciones para nuestra inserción en comunidades de práctica (Lave y Wenger (1998) y la creación de comunidades de aprendizaje, que han avanzado una agenda de investigación neurocognitiva en Puerto Rico.

Además de artículos y presentaciones en foros locales e internacionales, del Proyecto EFEL salieron cinco disertaciones doctorales y una tesis de maestría en las que se utilizaron la Teoría PASS y el CAS. Las autoras de estos trabajos se incorporaron a la academia en diversas instituciones de educación superior en Puerto Rico y en el extranjero, y han dado continuidad al proyecto. Destaca el trabajo de Mary Annette Moreno Torres (quien fue Coordinadora del Proyecto EFEL y es autora y co-autora de varios trabajos citados en este artículo). Ella ha continuado un vínculo de estrecha colaboración con Jack Naglieri y Tulio Otero, como demuestra su participación en la traducción del CAS 2 y en el manual de intervenciones para docentes y padres. Al mismo tiempo, ha servido de mentora y estimulado el trabajo de investigación de autoras y autores de trabajos incluidos en los volúmenes antes mencionados de la Revista Puertorriqueña de Psicología (Rodríguez Arocho y Moreno Torres, 2018). Luego de mi jubilación en 2011, ella ha asumido la mentoría y el liderato de una nueva generación de investigadores, y yo he reorientado mis intereses a la formación docente, la pedagogía crítica y los nuevos desarrollos teóricos en el enfoque histórico-cultural.

Puede decirse que el PREP y el Manual de intervenciones para uso en la escuela y en el hogar son las dos aplicaciones de la Teoría PASS donde es más evidente la mediación pedagógica. Sin embargo, la forma en que se ha utilizado el CAS (en sus dos versiones) permite argumentar que cumple una doble función: es una prueba para evaluar funcionamiento cognitivo, pero su uso se ha orientado a la identificación de debilidades y fortalezas con el propósito expreso de orientar intervenciones psicoeducativas.

Las investigaciones en los dos volúmenes mencionados cubren un amplio espectro de temas. El trabajo de Torres, Moreno Torres y Otero (2018) detalla la traducción y adaptación del CAS 2. La investigación de Moreno Torres, Díaz Flores, Bermonti Pérez y Rodríguez Arocho (2018) realiza una comparación entre los perfiles de 50 niños y niñas entre las edades de 6 a 11 años con diagnóstico de Trastorno por Déficit de Atención-Tipo combinado en la Escala Weschler de Inteligencia para Niños-Versión Puerto Rico y el CAS. Aunque ambas pruebas discriminaron entre este grupo y el grupo control, el examen de los resultados intragrupo sugiere que el CAS identifica mejor las características asociadas al 
trastorno. Esta precisión en la identificación de características facilita el diseño de intervenciones educativas que se enfoquen en ellas. Se han realizado investigaciones con otras poblaciones identificadas con condiciones especiales como estudiantes superdotados (Díaz Flores, González González y Moreno Torres, 2018), estudiantes en el espectro autista (Pérez Acevedo y Dávila Marrero, 2019) y estudiantes con problemas de aprendizaje, particularmente en la lectura (Cordero Arroyo, González González, Bermonti Pérez y Moreno Torres, 2018).

Las investigaciones mencionadas utilizan el CAS en contextos escolares con poblaciones identificadas con alguna condición. Dos investigaciones se enfocan en poblaciones típicas. Una de ellas (Báez Ávila, 2018) analiza la función de planificación con jóvenes jugadores de baloncesto, quienes ejecutaron mejor que el grupo control en todos los parámetros de esa función. La otra investigación (Ortiz Ortiz, 2019) aplica la teoría PASS y el CAS en un estudio que utiliza el videojuego como instrumento de mediación en aprendizaje de destrezas de lecturas y habilidades cognitivas en estudiantes que aprenden el idioma inglés. Un tercer trabajo (Sepúlveda-Miranda, Otero y Moreno Torres, 2018) examina consideraciones legales en los procesos de evaluación a niños y niñas con desórdenes específicos de aprendizaje en contextos educativos en Puerto Rico y Estados Unidos. El trabajo analiza investigaciones en el campo de la evaluación con esta población, y argumenta que el CAS ofrece una mejor alternativa que las pruebas tradicionales para enfrentar diversidad cultural y lingüística. Estas investigaciones destacan el valor del CAS para el desarrollo de intervenciones de educación cognitiva en diversos procesos. Procede ahora examinar con mayor detalle las investigaciones que realizaron intervenciones particulares de mediación pedagógica.

Las investigaciones de Báez (2019) y Medina (2018) se originan en sus disertaciones doctorales en el Proyecto EFEL. Ambas realizaron estudios para probar la efectividad del PASS Reading Enhancement Program (PREP). Hicieron una adaptación de la traducción de la prueba realizada en España y utilizaron el CAS como medida pre y post intervención. Medina utilizó el PREP como instrumento de mediación pedagógica en un grupo de 5 niños y niñas con problemas de lectura y diagnóstico del Trastorno de Déficit de Atención con Hiperactividad. Ambos desórdenes tienen una alta incidencia en Puerto Rico, según estadísticas de fuentes gubernamentales. En su examen de la literatura, la investigadora destaca algunas de las funciones cognitivas comprometidas en ambos desórdenes, como problemas de atención, memoria de trabajo, velocidad y capacidad de procesamiento de 
información, particularmente la relacionada con el lenguaje. La intervención, que va orientada a fortalecer estas funciones, se realizó individualmente, aunque es posible realizarla en grupo. Como se mencionó antes, el programa se apoya fuertemente en la relación y colaboración entre las partes, y el énfasis es en la enseñanza de estrategias para la solución de problemas enfrentados en la solución de las tareas.

El programa fue aplicado individualmente en interacciones que fluctuaron entre 10 y 15 semanas en función de las características y necesidades de las personas participantes. Cada sesión tenía dos periodos de 45 minutos de duración. Además de utilizar el CAS, Medina (2018) utilizó en la pre y la post prueba dos subpruebas de Batería de Aprovechamiento Woodcock Muñoz-Revisada para evaluar el rendimiento de lectura de las personas participantes. Las subpruebas fueron las de identificación de letras y palabras, además de comprensión de textos. Los resultados mostraron que la mediación pedagógica fue efectiva tanto en el fortalecimiento de destrezas cognitivas como en la ejecución lectora (Medina, 2018). La función cognitiva con mayores cambios fue el procesamiento sucesivo.

La investigación de Báez (2019) también se enfoca en probar la efectividad del PREP como programa de educación cognitiva, pero su alcance es más específico. Se enfocó en las tareas puente de este programa de educación cognitiva. La intervención se realizó con tres niños y una niña en escuela elemental. Además de utilizar los mismos instrumentos que Medina (2018) para evaluar funcionamiento cognitivo y destrezas de lectura, el CAS y las subpruebas mencionadas de la Batería de Aprovechamiento Woodcock Muñoz-Revisada, el estudio de Báez introdujo dos instrumentos para una mirada cualitativa. El primero fue un registro anecdótico, un instrumento básico de anotación de observaciones no sistematizadas realizadas por una observadora. Su propósito fue crear una descripción redactada como anécdota que registró eventos que destacan una conducta o comportamiento en el contexto en que ocurre. En última instancia, el registro es un relato de la relación sujeto-contexto. El segundo instrumento fue un cuestionario posterior a la intervención de cuatro preguntas que fue administrado a las madres de las personas participantes para conocer su valoración de la intervención educativa y sus resultados.

Las tareas puente del PREP fueron realizadas en 15 sesiones. La investigadora dirigió las sesiones de mediación pedagógica y contó con la asistencia de una estudiante de postgrado del Departamento de Psicología de la Universidad de Puerto Rico. Utilizó las tareas puente para procesamiento sucesivo: 1) relación entre partes, 2) unión de figuras, 3) secuenciación memorizada de matrices, 4) conexión de letras y 5) secuenciación 
memorizada de elementos. También las tareas puente para procesamiento simultáneo: 1) recuerdo de matrices, 2) verificación de significados, 3) seguimiento de pistas, 4) construcción de estructuras y 5) asociación de estructuras. Los resultados en los instrumentos estandarizados evidencian mejoramiento en las funciones de procesamiento simultáneo y sucesivo, así como en la lectura.

Es pertinente señalar que también se observó mejoramiento en atención y planificación. Un dato interesante es que los instrumentos cualitativos, además del mejoramiento en estas capacidades, informan cambios conductuales y afectivos en las personas participantes. La investigadora resalta el potencial del PREP para propiciar el aprendizaje de estrategias y el uso del lenguaje hablado. Dado que en cada tarea se requiere que el o la participante verbalice mientras resuelve o lleva a cabo la tarea, se crean condiciones que favorecen la interacción durante la intervención y el aprendizaje (Báez, 2019).

Otra de las aplicaciones de la teoría PASS que ha sido objeto de investigación en Puerto Rico es el manual de intervenciones Helping children learn: Interventions handhouts for use in school and at home (Naglieri y Pickering, 2010). Cordero Arroyo, González González, Bermonti-Pérez y Moreno Torres (2018) investigaron el impacto de algunas de las intervenciones orientadas al mejoramiento de funciones asociadas a la lectura, específicamente, las relacionadas con el procesamiento sucesivo evidenciadas en el deletreo. Utilizaron un diseño de caso único, que implica tomar diversas medidas de ejecución de un mismo sujeto en un plazo de tiempo y donde el sujeto sirve como su propio control. En este caso, el objetivo era mejorar destrezas de deletreo. Para ello, se desarrolló una intervención que utilizó dos de las guías contenidas en el referido manual: 1) enseñanza sobre sobre planificación y 2) estrategias para deletrear. La mediación pedagógica está orientada a enseñar a pensar sobre lo que se está haciendo. La idea es promover metacognición con respecto a las estrategias utilizadas en la realización de la tarea y su efectividad. Para lograr esto, al momento de implementar esta intervención, se solicita a las personas participantes responder a preguntas relacionadas a los ejercicios de deletreo.

En este estudio la modalidad de la intervención fue individual. Como en el estudio anterior, la investigadora realizó la intervención, en este caso con la asistencia de estudiantes de pregrado en psicología. Cada participante tuvo dos sesiones por semana, cada una de una hora, con una duración de dos meses y tres semanas. En cada sesión el estudiantado tuvo 30 minutos para completar las medidas de deletreo y realizar actividades 
recreativas o recibir la intervención. Completaron cuatro semanas de línea de base (dos antes y dos después de la intervención) y siete semanas de intervención, para un total de once semanas. Los resultados muestran que todas las personas participantes mejoraron su ejecución en, por lo menos, tres de las cuatro funciones cognitivas evaluadas luego de participar de la mediación pedagógica. Los mayores cambios se observaron en el procesamiento sucesivo y en la planificación. Junto a estos cambios en funcionamiento cognitivo, también se observó mejoramiento en destrezas de lectura, particularmente, las que requieren dominio de aspectos mecánicos y entendimiento de las unidades básicas del lenguaje escrito (fonemas y grafemas).

En un estudio similar, Bermonti-Pérez, Cordero-Arroyo y Moreno (2018) se enfocaron en probar una versión computadorizada de la intervención para mejorar procesos cognitivos implicados en el deletreo. Usaron la intervención llamada Ordenamiento de Letras para Deletrear en el manual de intervenciones de Naglieri y Pickering (2010) antes descrito. El propósito de esta es desarrollar la destreza de deletreo al enfocar la atención estudiantil en las características sucesivas de las palabras, lo que debe impactar su habilidad para decodificar letras en sonidos, reconocer patrones visuales en las palabras y la capacidad de memorizar el orden de las letras. La metodología del estudio fue cualitativa e incluyó una revisión de expertos y entrevistas semiestructuradas a las personas participantes para explorar tanto el proceso como el resultado. Los estudiantes y las estudiantes evaluaron como útil la intervención. Aunque algunos señalaron falta de flexibilidad en el sistema computadorizado. Los resultados generales apuntan a la viabilidad de uso con escolares de tercer y cuarto grados.

En resumen, las investigaciones realizadas para probar la efectividad de mediación pedagógica en la modificación cognitiva la respaldan como una actividad que puede mejorar funciones importantes en la realización de actividades como planificar, atender y procesar información secuencial y simultáneamente. Estas actividades se correlacionan altamente con el aprovechamiento escolar, lo que resalta la centralidad de la mediación pedagógica en la educación cognitiva. Estas investigaciones se suman a otras realizadas en Puerto Rico, orientadas a integrar psicología y educación en teoría, investigación y práctica. Una muestra de ello puede observarse en el volumen de la Revista Puertorriqueña de Psicología dedicado a psicología y educación, en el que aparecen otras muestras de mediación pedagógica (Rodríguez Arocho y Bernal, 2016). 


\section{Conclusiones}

La evaluación psicoeducativa se realiza desde diversas perspectivas teóricas. Estas perspectivas descansan en premisas filosóficas que implica visiones de mundo y formas de abordar la ciencia como actividad humana. La idea de la cognición como una propiedad individual predeterminada e inalterable enfrenta, en la actualidad, ideas alternativas que conceden a la educación un rol fundamental en formación y desarrollo de funciones cognitivas. Nuevas teorías sobre el funcionamiento intelectual, nuevos desarrollos tecnológicos para estudiar ese funcionamiento, reclamos sociales por mayor justicia y equidad en las prácticas y usos de la evaluación psicoeducativa ofrecen la posibilidad de mejorar situaciones de desventaja para estudiantes en todos los niveles escolares, pero particularmente en los más tempranos. El programa de investigación que he examinado respalda la mediación pedagógica como estrategia de educación cognitiva para mejorar la ejecución en evaluaciones psicoeducativas. El recuento realizado en este trabajo puede ayudar a colocar tanto la evaluación psicoeducativa como la mediación pedagógica actuales en perspectiva histórica y sociocultural y, desde ahí, pensar su futuro. Ese futuro implica formas dinámicas de evaluación y reflexión continua sobre la práctica profesional y sus consecuencias sobre los sujetos que son evaluados. Avanzar hacia ese futuro demanda más investigación. Los estudios presentados son un punto de partida, pero queda pendiente la tarea de ampliar el conocimiento con respecto a los resultados derivados de la implementación de la mediación pedagógica como estrategia de educación cognitiva para mejorar la ejecución en evaluaciones psicoeducativas.

\section{Referencias}

Akutina, Tatiana. (2003). L. S. Vygotsky and A. R. Luria: Foundations of Neuropsychology. Journal of Russian \& East European Psychology, 41(4), 159190, DOI: 10.2753/RPO1061-0405410304159

Andrews, Jac., Saklofske, Donald H. y Janzen Henry L. (Eds.) (2001). Handbook of psychoeducational assessment: Ability, achievement and behavior in children. New York: Academic Press.

Ardila, Alfredo. (2018). Historical development of human cognition: A cultural-historical perspective. Singapore: Springer. DOI 10.1007/978-981-10-6887-4

Báez Ávila, Loggina. (2018). Procesos de planificación en adolescentes balocelistas. Revista Puertorriqueña de Psicología, 29(2), 232-246. Recuperado de http://www.ojs.repsasppr.net/index.php/reps/article/view/425 
Báez, María. (2019). Estudio para explorar la posibilidad de modificación neurocognitiva: Aplicación del Modelo PASS. Revista Puertorriqueña de Psicología, 30(1), 140-154. Disponible en http://www.ojs.repsasppr.net/index.php/reps/article/view/434/614

Bermonti-Pérez, Mario., Cordero-Arroyo, Giselle y Moreno Torres, Mary Annette. (2018). Evaluación de la versión computadorizada de una intervención cognitiva para mejorar procesamiento sucesivo y deletreo. Revista Puertorriqueña de Psicología, 29 (2), 288231. Disponible en http://www.ojs.repsasppr.net/index.php/reps/article/view/431/592

Bermonti-Pérez, Mario., Díaz-Flores, Wilmarie., Moreno-Torres, Mary Annette. y Rodríguez Arocho, Wanda C. (2014). Concepciones sobre Inteligencia, y su relación con habilidades lingüísticas en niños y niñas de habla hispana. Salud y Conducta Humana, 1(1), 1-12. Recuperado de https://static1.squarespace.com/static/50c39c53e4b097533b3492dd/t/5342c083e4b022 33d7457fd7/1396883587578/1 Bermonti D\%C3\%ADaz Moreno Rodr\%C3\%ADguez 2014.pdf

Cole, Michael. (1996). Cultural psychology: A once and future discipline. Cambridge, MA: Harvard University Press.

Cole, Michael., Levitin, Karl. y Luria, Alexander. (2006). The autobiography of Alexander Luria: A dialogue with The making of mind. New Jersey: Lawrence Erlbaum Associates. DOI https://doi.org/10.4324/9781315799353

Cordero-Arroyo, Giselle., González-González, Manuel., Bermonti-Pérez, Mario. y Moreno Torres, Mary Annette. (2018). Intervención neuropsicológica pasa estudiantes con inatención, hiperactividad y dificultades en lectura. Revista Puertorriqueña de Psicología, 29(2), 259-268. Recuperado de http://www.ojs.repsasppr.net/index.php/reps/article/view/427

Dafermos, Manolis. (2014). Vygotsky's analysis of the crisis of psychology: Diagnosis, treatment and relevance. Theory \& Psychology, 24(2), 147-165. https://doi.org/10.1177/0959354314523694

Dafermos, Manolis. (2018). Rethinking cultural-historical theory: A dialectical perspective to Vygotsky. Singapore: Springer. DOI: https://doi.org/10.1080/10749039.2020.1779750

Daniels, Harry. (2001). Vygotsky and pedagogy. New York: Routledge. DOI: https://doi.org/10.4324/9781315617602

Daniels, Harry. y Hedegaard, Mariane (Eds.). (2011). Vygotsky and special needs education: Rethinking support for children and schools. New York: Continuum Education Publishing Group. DOI: https://doi.org/10.1080/09500790.2011.588019

Das, Jagannath Prasad. (2000). PREP: A cognitive remediation program in theory and practice. Developmental Disabilities Bulletin, 28(2), 83-96.

Das, Jagannath Prasad., Naglieri, Jack. y Kirby, John R. (1994). Assessment of cognitive processes: The PASS theory of intelligence. Allyn \& Bacon: Boston, MA. 
Dazinger, Kurt. (1997). Naming the mind: How psychology found its language. Thousand Oaks, CA: Sage Publications. DOI: http://dx.doi.org/10.4135/9781446221815

Díaz Flores, Wilmarie., González González, Manuel., y Moreno Torres, Mary Annette. (2018). Desempeño en el Cognitive Assessment System 2- español de estudiantes superdotados. Revista Puertorriqueña de Psicología, 29(2), 316-330. Recuperado de http://www.ojs.repsasppr.net/index.php/reps/article/view/429

Dombrowski, Stefan C. (2015). Psychoeducational assessment and report writing. New York: Springer. Recuperado de https://link.springer.com/book/10.1007/978-1-4939-1911-6

Garrido Laparte, María de los Ángeles. (1999). Programa PREPPRDA: traducción adaptación, validación y primera experiencia. En J. P. Das, M. A. Garrido Laparte, M. González, C. Timoneda y F. Pérez-Alvarez (Eds.), Dislexia y dificultades de lectura (pp. 211-239). Madrid: Editorial Paidós.

González Rey, Fernando Luis. (2009). Epistemología y ontología un debate necesario para la psicología de hoy. Diversitas: Perspectivas en Psicología, 5(2), 205-224. DOI: 10.15332/s1794-9998.2009.0002.01

Guadarrama González, Pablo. (2018). Para que sirve la epistemología a un investigador y un profesor. Bogotá: Cooperativa Editorial Magisterio.

Haywood, H. Carl. (2013). What is cognitive education? A view from 30,000 feet. Journal of Cognitive Education and Psychology, 12(1) 1-18. Recuperado de https://connect.springerpub.com/content/sgricep/12/1/26

Kozulin, Alex. (2000). Instrumentos psicológicos: La educación desde la perspectiva sociocultural. Buenos Aires: Paidós.

Kozulin, Alex. (2003). Psychological tools and mediated learning. En Alex Kozulin, Boris Gindis, Vladimir Agueyev y Susan Miller (Eds.), Vygotsky's educational theory in cultural context (pp. 15-38). New York: Cambridge University Press. DOI: https://doi.org/10.1017/CBO9780511840975.003

Kozulin, Alex. (2014). Dynamic assessment in search of identity. En A. Yasnitsky, R. Van der Veer y M. Ferrari (Eds.), The Cambridge handbook of cultural-historical psychology (pp. 126-147). New York: Cambridge University Press. DOI: https://doi.org/10.1017/CBO9781139028097.008

Lave, Jean. y Wenger, Etienne. (1991). Situated learning: Legitimate, peripheral participation. New York: Cambridge University Press. DOI: https://doi.org/10.1017/CBO9780511815355

León León, Giselle (2014). Aproximaciones a la mediación pedagógica. Revista Calidad en la Educación Superior, 5(1), 136-155. Recuperado de https://dialnet.unirioja.es/descarga/articulo/5580842.pdf 
Luria, Alexander R. (1961). The role of speech in the regulation of normal and abnormal behavior. New York: Pergamon Press. DOI: 10.1126/science.134.3485.1063

Luria, Alexander R. (1973). The working brain. London: Penguin Books. DOI: https://doi.org/10.1176/ajp.133.3.349

Luria, Alexander R. (1976). Cognitive development: Its cultural and social foundations. Cambridge, MA: Harvard University Press.

Luria, Alexander R. (1982). Language and cognition. New York: John Willey and Sons.

Medina, Nilda. (2018). Cognitive Modification in students with reading problems and ADHDCT. Revista Puertorriqueña de Psicología, 29(2), 302-315. Recuperado de http://www.ojs.repsasppr.net/index.php/reps/article/view/433

Mitjáns Martínez, Albertina. (2009). La perspectiva histórico-cultural y la educación especial: Contribuciones iniciales y desarrollos actuales. Actualidades en Investigación Educativa, 9(Especial). DOI 10.15517/aie.v9i4.9517

Moll, Luis C. (Ed.). (1990). Vygotsky and education: Instructional implications and applications of sociohistorical psychology. Cambridge: Harvard University Press. DOI: https://doi.org/10.1017/CBO9781139173674

Moll, Luis C. (2014). L.S. Vygotsky and education. New York: Routledge. DOI: https://doi.org/10.1080/00071005.2015.1006957

Moreno Torres, Mary Annette., Díaz Flores, Wilmarie., Bermonti-Pérez, Mario. y Rodríguez Arocho, Wanda C. (2018). Comparación de Perfiles del TDAH-TC en la EIWN-R-PR y el CAS. Revista Puertorriqueña de Psicología, 29(2), 224-237. Recuperado de http://www.ojs.repsasppr.net/index.php/reps/article/view/438

Naglieri, Jack A. y Das, Jagannath Prasad. (1997). Das-Naglieri Cognitive Assessment System. Itasca, IL: Riverside Publishing. https://doi.org/10.1177/073428299000800309

Naglieri, Jack A., Das, Jagannath Prasad. y Goldstein, Sam. (2012). En Dawn Flanagan and Patti L. Harrison (Eds.), Contemporary intellectual assessment: Theories, tests, and issues (3rd ed.; pp. 178-191). New York: Guilford. DOI: http://dx.doi.org/10.1037/t27677-000

Naglieri, Jack A., and Otero, T. M. (2011). Cognitive Assessment System: Redefining Intelligence From Neuropsychological Perspective. En A. Davis (Ed.). Handbook of Pediatric Neuropsychology (pp. 320-332). New York: Springer Publishing. DOI: http://dx.doi.org/10.1037/t15176-000

Naglieri, Jack A. y Otero, Tulio. (2017). Essentials of CAS 2 Assessment. New York: John Willey.

Naglieri, Jack, A. y Otero, Tulio. (2018a). Essentials of CAS2 Assessment. New York: Wiley. 
Naglieri, Jack A. y Otero, Tulio (2018b). The Cognitive Assessment System: From theory to practice. In D. P. Flanagan \& E. M. McDonough (Eds.), Contemporary intellectual assessment: Theories, tests, and issues (p. 452-485). The Guilford Press.

Naglieri, Jack A., Moreno, Mary Annette., and Otero, Tulio. (2017). Cognitive Assessment System 2: Español. Austin, TX: ProEd Inc.

Naglieri, Jack A. y Pickering, Eric. (2010). Helping children learn: Intevention handhouts for use in school and home. New York: Brookes Publishers. DOI: 10.1002/pits.20034

Ortiz-Ortiz, Yamil. (2019). A neurocognitive video game intervention effects on the reading skills and cognitive abilities of English language learners: Pilot study. Revista Puertorriqueña de Psicología, 30(1), 122-137. Recuperado de http://www.ojs.repsasppr.net/index.php/reps/article/view/436

Pérez Acevedo, Sacha y Dávila Marrero, Elixmair. (2019). Perfil neurocognitivo de jóvenes diagnosticados el trastorno del espectro autismo altamente funcional. Revista Puertorriqueña de Psicología, 30(1), 156-158. Recuperado de http://www.ojs.repsasppr.net/index.php/reps/article/view/432

Rodríguez Arocho, Wanda C. (2006). Estudio de los procesos cognitivos en Puerto Rico: Antecedentes, actualidades y perspectivas. Revista Puertorriqueña de Psicología, $\begin{array}{lcc}17(1) & 517-549 . & \text { Recuperado } \\ \text { http://www.ois.repsasppr.net/index.php/reps/article/view/514 }\end{array}$

Rodríguez Arocho, Wanda C. (2003). La relación entre funciones ejecutivas y lenguaje: Una propuesta para estudiar su relación. Perspectivas Psicológicas, 3-4, 41-48. Recuperado de http://pepsic.bvsalud.org/pdf/pp/v3-4/v3-4a05.pdf

Rodríguez Arocho, Wanda C. (2004). Desarrollo de funciones ejecutivas y su relación con el lenguaje: En busca de un enfoque integrado para su investigación. (2004). Ciencias de la Conducta, 19(1), 1-18. Recuperado de http://bookshelf.albizu.edu/pdf/2004.php

Rodríguez Arocho, Wanda C. (2011). Aprendizaje, desarrollo y evaluación en contextos escolares: Consideraciones teóricas y prácticas desde el enfoque histórico-cultural. Actualidades Investigativas en Educación, 11(1), 1-36. DOI 10.15517/aie.v11i1.10168

Rodríguez Arocho, Wanda C. (2015). Reflexividad histórica, problematización e indagación dialógicas como herramientas para repensar el concepto vygotskiano de zona de desarrollo próximo. Revista Puertorriqueña de Psicología, 26(1), 10-24. Recuperado de https://dialnet.unirioja.es/servlet/articulo?codigo=5891741

Rodríguez Arocho, Wanda C. (2018). Tecnologías de la información y la comunicación: nuevas configuraciones mentales y sus implicaciones para la educación. Revista de Psicología, 27(1), 1-2. DOI: 10.5354/0719-0581.2018.50751 
Rodríguez Arocho, Wanda C. y Bernal, Guillermo. (2016). Psicología, educación y contextos de práctica: Una introducción al número especial. Revista Puertorriqueña de Psicología, 27(2), 200-204. Recuperado de http://www.ojs.repsasppr.net/index.php/reps/article/view/286/287

Rodríguez Arocho, Wanda C. y Moreno Torres, Mary Annette. (2018). En busca de justicia y equidad en la evaluación cognitiva: Aplicaciones de la Teoría PASS y del Cognitive Assessment System. Revista Puertorriqueña de Psicología, 29(2), 216-222. Recuperado de http://www.ojs.repsasppr.net/index.php/reps/article/view/525/587

Rodríguez Arocho, Wanda C. y Alom Alemán, Antoinette. (2009). El enfoque sociocultural en el diseño y construcción de una comunidad de aprendizaje. Actualidades en Investigación Educativa, 9(Especial). DOI 10.15517/aie.v9i4.9520

Schraw, Gregory. (2013). Conceptual integration and measurement of epistemological beliefs in educational research. ISRN Education. http://dx.doi.org/10.1155/2013/327680

Sepúlveda Miranda, Paola M., Otero, Tulio. y Moreno Torres, Mary Annette. (2018). Moving toward social justice for Hispanic children using a neurocognitive method for the assessment of SLD. Revista Puertorriqueña de Psicología, 29(2), 270-286. Disponible en http://www.ojs.repsasppr.net/index.php/reps/article/view/435

Valsiner, Jaan. (2013). A guided science: History of psychology in the mirror of tis making. New Brunswick: Transaction Publishing. DOI: https://doi.org/10.1002/jhbs.21615

Vygotski, Lev S. (1931). Historia del desarrollo de las funciones psíquicas superiores. En $L$. S. Vygotsky: Obras Escogidas. (Tomo III; pp. 10-325). Madrid. Aprendizaje Visor.

Vygotski, Lev S. (1991). El significado histórico de la crisis de la psicología. En L. S. Vygotski: Obras Escogidas (Tomo I; pp. 275-413). Madrid: Aprendizaje Visor.

Vygotski, Lev S. (1994). Tool and symbol in child development. En R. Van der Veer y J. Valsiner (Eds.), The Vygotsky reader (pp. 99-174). New York: Blackwell.

Vygotsky, Lev S. (1934). Pensamiento y lenguaje. En L. S. Vygotsky: Obras Escogidas (Tomo II; pp. 9-348). Madrid. Aprendizaje Visor.

Vygotsky, Lev S. y Luria, Alexander, R. (1930). Tool and symbol in child development. En René Van der Veer 7 Jann Valsiner (Eds.), The Vygotsky reader (pp. 99-174). New York: Blackwell.

Wertsch, James V. (1991). Voces de la mente: Un enfoque sociocultural para el estudio de la acción mediada. Madrid: Aprendizaje Visor.

Yasntisky, Anton., Van der Veer, René., Aguilar, Efraín. y García, Luciano Nicolás (Eds.). (2016). Vygotski revisitado: Una historia de su contexto y legado. Buenos Aires: Miño y Dávila Editores. 
Revista indizada en
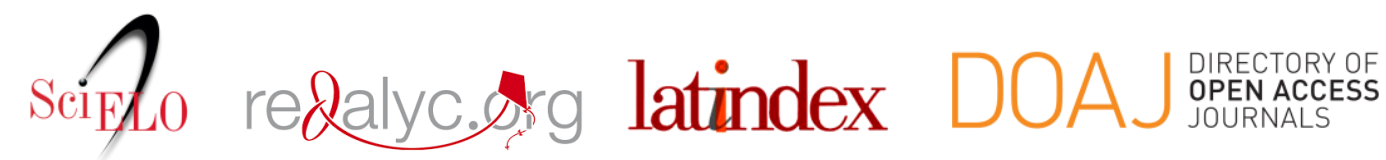

Distribuida en las bases de datos:

- Dialnet

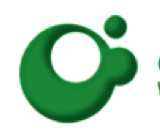
SHERPA/RøMEO

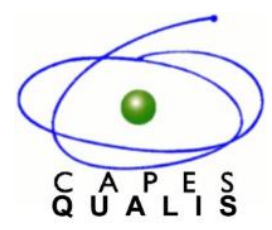

MIAR 